\title{
DESEMPENHO AGRONÔMICO DE LIMA ÁCIDA TAHITI EM COMBINAÇÃO COM DIFERENTES PORTA-ENXERTOS EM CAPIXABA, ACRE
}

Maria Júlia da Silva Rodrigues ${ }^{1}$, Romeu de Carvalho Andrade Neto ${ }^{2}$, Lauro Saraiva Lessa $^{3}$, Eduardo Augusto Girardi ${ }^{4}$, Walter dos Santos Soares Filho ${ }^{4}$

${ }^{1}$ Engenheira Agrônoma, D. Sc. em Produção Vegetal, Universidade Federal do Acre, julia.agro32@gmail.com

${ }^{2}$ Engenheiro Agrônomo, D. Sc., pesquisador da Embrapa Acre, Rio Branco, AC,

${ }^{3}$ Engenheiro Agrônomo, D. Sc., analista da Embrapa Acre, Rio Branco, AC,

${ }^{4}$ Engenheiro Agrônomo, D. Sc., pesquisador Embrapa Mandioca e Fruticultura, Cruz das Almas-BA

Recebido em: 22/09/2018 - Aprovado em: 23/11/2018 - Publicado em: 03/12/2018 DOI: 10.18677/EnciBio 2018B30

\section{RESUMO}

No Acre a produção de lima ácida Tahiti é pequena e não atende à demanda local, apesar de possuir condições climáticas adequadas ao seu cultivo. Para se obter melhor desempenho produtivo desta cultura é necessária a utilização de portaenxertos que apresentem adaptação às características edafoclimáticas da região e compatibilidade com a copa estudada. Assim, o objetivo desse estudo foi avaliar o desempenho de lima ácida Tahiti sobre diferentes porta-enxertos de citros em Capixaba, Acre. O experimento foi conduzido em área de produtor, no município de Capixaba, Acre. Avaliou-se a limeira ácida Tahiti (C. latifolia (Yu. Tanaka.), sobre dez porta-enxertos introduzidos ou obtidos pelo Programa de Melhoramento Genético de Citros da Embrapa Mandioca e Fruticultura: citrandarins Indio, Riverside e San Diego, limoeiro Cravo Santa Cruz, tangerineira Sunki Tropical e os híbridos TSKFL x CTTR - 013, LVK x LCR - 038, LVK x LVA-009, TSK x CTSW - 041 e TSKFL $x$ CTTR - 017. O delineamento experimental adotado foi em blocos ao acaso, com dez tratamentos (porta-enxertos), três repetições e duas plantas por parcela. Avaliaramse características vegetativas e de qualidade dos frutos. Houve efeito dos portaenxertos sobre o comportamento da variedade copa. Até o sexto ano de plantio, os citrandarins Indio, Riverside e San Diego, tangerineira Sunki Tropical e limoeiro Cravo Santa Cruz proporcionaram melhor desempenho agronômico à lima ácida Tahiti.

PALAVRAS- CHAVE: Amazônia Sul Ocidental, Citrus latifolia, Poncirus trifoliata (L.) Raf.

\section{AGRONOMIC PERFORMANCE OF TAHITI ACID LIME IN COMBINATION WITH DIFFERENT GASKETS IN CAPIXABA, ACRE}

\begin{abstract}
The Tahiti acid lime production is small in Acre and does not meet local demand, despite adequate climatic conditions for its growing. In order to obtain bigger production, it's necessary the use of rootstocks that are adapted to the region and adaptability to the cup. Thus, the objective of this work was to evaluate Tahiti acid
\end{abstract}


lime performance over diferente rootstocks in Capixaba, Acre. Tahiti acid lime ( $C$. latifolia (Yu. Tanaka.) was evaluated over tem rootstocks introduced or obtained by Citrus genetic improvement program of Embrapa Mandioca e Fruticultura: Indio, Riverside and San Diego citrandarins, Cravo Santa Cruz lemon, Sunki Tropical tangerine and TSKFL $\times$ CTTR - 013, LVK $\times$ LCR - 038, LVK $\times$ LVA-009, TSK $x$ CTSW - 041 e TSKFL $\times$ CTTR - 017 hybrids. The experimental design used was completely randomized blocks with tem treatments (rootstocks), two replications and two plants per plot. Vegetative characteristics and fruit quality were evaluated. The Tahiti scion were influenced by rootstocks. Until sixth year of planting, Indio, Riverside e San Diego citrandarins, Sunki Tropical tangerine and Cravo Santa Cruz lemon provide to Tahiti acid lime better agronomic performance.

KEYWORDS: South Western Amazon, Citrus latifolia, Poncirus trifoliata (L.) Raf.,

\section{INTRODUÇÃO}

A lima ácida Tahiti [Citrus latifolia (Yu. Tanaka) Tanaka] é conhecida e comercializada no mercado brasileiro como limão, razão pela qual também é chamada de limão Tahiti. Apesar de o Brasil ser o maior produtor mundial de laranja e exportador de suco dessa fruta, a limeira ácida Tahiti se destaca na citricultura de exportação nacional in natura (IBRAF, 2015). O Brasil produz anualmente 1,2 milhões de toneladas, correspondendo à aproximadamente $8 \%$ da produção mundial de limões/lima ácida, e ocupa o quinto lugar na produção mundial dessa fruta, superado pelo México, Índia, China e Argentina (FAO, 2018). O espaço conquistado no mercado externo é devido, principalmente, ao maior consumo de caipirinha e ao uso da fruta para adornar pratos e bebidas, em substituição aos limões 'Verdadeiros'.

O Brasil, em 2017 colheu mais de 48 mil hectares de limeira ácida Tahiti, sendo São Paulo, com 25.869 ha colhidas, o principal produtor (IBGE, 2018). O Norte do país possui um imenso potencial para a produção de frutos cítricos, devido à aptidão climática para o seu cultivo, além de apresentar preços compensadores. Apesar das condições favoráveis, a produtividade média da lima ácida Tahiti é baixa $\left(17,4 \mathrm{t} \mathrm{ha}^{-1}\right)$ se comparada a de outras regiões, como o sudeste brasileiro cuja produtividade média, em 2017, foi de 34,9 tha ${ }^{-1}$ (IBGE, 2018). No Acre a média de produtividade obtida em 2017 foi de 15,4 tha-1 , ainda aquém dos principais polos produtores.

As plantas cítricas para formação de pomares comerciais é composta por dois indivíduos unidos por meio da enxertia: o porta-enxerto, responsável pela sustentação e nutrição da planta e o enxerto que é encarregado de formar a copa e a produção de frutos.

O porta-enxerto desempenha um papel fundamental em combinação com a cultivar de copa e influencia várias características das plantas, como crescimento, desenvolvimento, precocidade de produção, tolerância a fatores abióticos (seca) e a abióticos (pragas e doenças), assim como no desenvolvimento, maturação, qualidade e rendimento de frutos (CARVALHO et al., 2016).

Apesar de ter ocorrido estudo no sentido de definir o melhor porta-enxerto para a limeira ácida Tahiti (LEDO et al., 2008), ainda persiste carência de informações em relação às combinações copa/porta-enxerto, pois, atualmente, apesar de induzir boas produções logo nos primeiros anos e mantê-las durante a vida útil do pomar, a combinação de Tahiti e o porta-enxerto de limoeiro Cravo (Citrus limonia Osbeck) tem vida útil reduzida devido à alta incidência de gomose de Phytophthora spp.

Assim, o objetivo desse estudo foi avaliar o desempenho de limeira ácida Tahiti [C. latifolia (Yu. Tanaka) Tanaka] sobre diferentes porta-enxertos de citros no 
municípi de Capixaba, Acre.

\section{MATERIAL E MÉTODOS}

O experimento foi conduzido em área de produtor, no município de Capixaba, Acre. O local do experimento está a $10^{\circ} 21^{\prime} 07,07^{\prime \prime}$ de latitude $S$ e 67'34'46,94" de longitude $\mathrm{O}$ à $179 \mathrm{~m}$ de altitude. $\mathrm{O}$ clima da região é do tipo $\mathrm{AWI}$ (quente e úmido), conforme a classificação de Köppen, com temperatura máxima de $31,76^{\circ} \mathrm{C}$, mínima de $19,50^{\circ} \mathrm{C}$, precipitação anual variando de 1.680 a $2.580 \mathrm{~mm}$ e umidade relativa de 79\% (AGRITEMPO, 2018). Os dados meteorológicos foram monitorados durante 0 período do experimento (Figura 1).

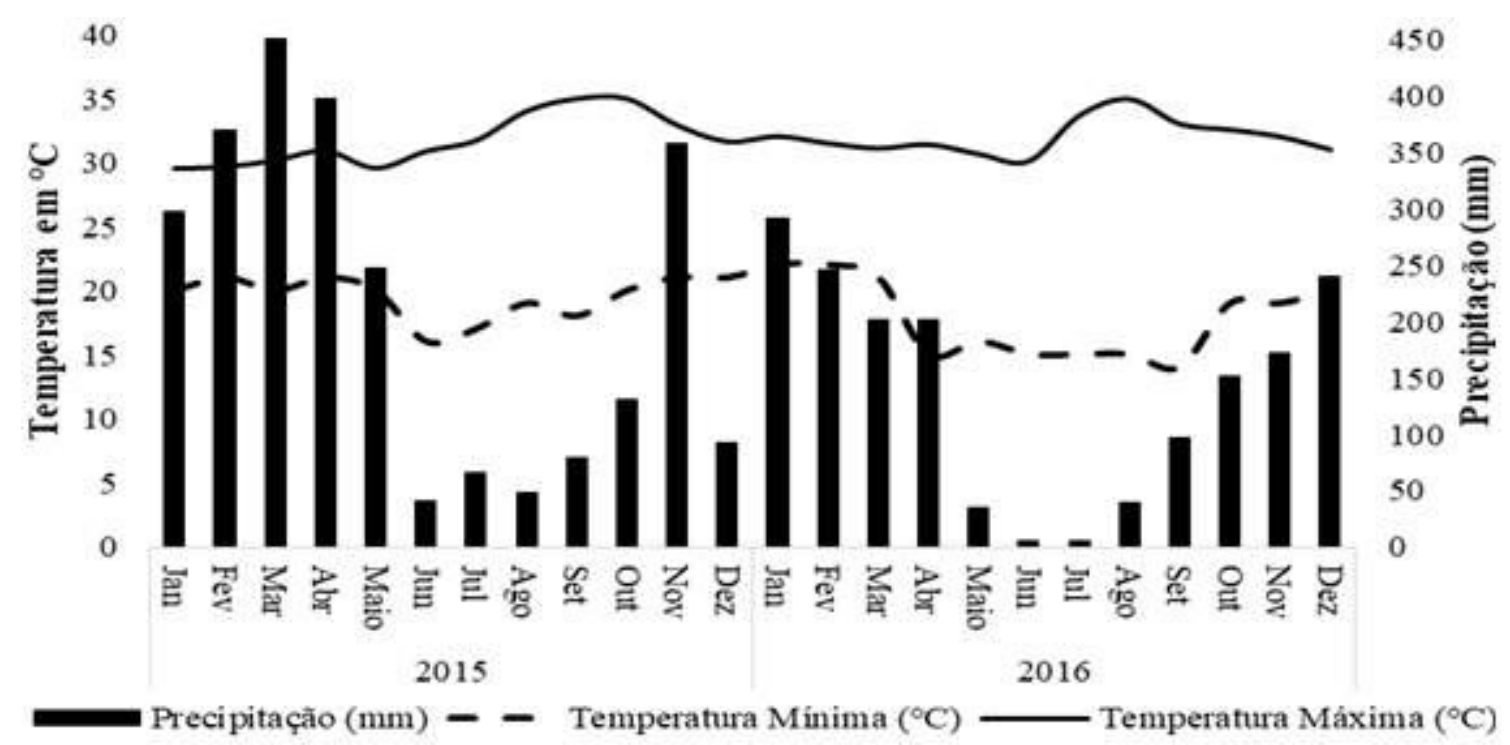

FIGURA 1 - Precipitação pluviométrica e temperatura do ar máxima e mínima mensal na área experimental. Capixaba-AC. 2015-2016

A área apresenta relevo plano tendendo à suave ondulado e com predominância de Argissolo Vermelho Distrófico típico de textura média argilosa, apresentando na camada superficial do solo de $0-20 \mathrm{~cm}$ os atributos químicos apresentados na tabela 1 .

TABELA 1 - Análise química do solo da área experimental, Capixaba, Acre, 2016

\begin{tabular}{ccccccccccccccc}
\hline $\begin{array}{c}\mathrm{pH} \\
\left(\mathrm{H}_{2} \mathrm{O}\right)\end{array}$ & $\mathrm{Ca}^{2+}$ & $\mathrm{Mg}^{2+}$ & $\mathrm{K}^{+}$ & $\mathrm{Na}^{+}$ & $\mathrm{H}+\mathrm{Al}^{2}$ & $\mathrm{Al}^{3+}$ & $\mathrm{SB}$ & $\mathrm{CTC}_{7,0}$ & $\mathrm{CTC}_{\text {ef }}$ & $\mathrm{P}$ & $\mathrm{P}_{\mathrm{rem}}$ & $\mathrm{V}$ & $\mathrm{Al}$ & $\mathrm{MO}$ \\
\hline 5,19 & 0,66 & 0,48 & 0,01 & 0,01 & 2,13 & 0,05 & 1,16 & 3,29 & 1,21 & 9,66 & 40,82 & 35,2 & 4,3 & 7,93 \\
\hline
\end{tabular}

MO: matéria orgânica

Avaliou-se a limeira ácida Tahiti (C. latifolia (Yu. Tanaka.), variedade local caipira, sobre dez porta-enxertos de citros introduzidos ou obtidos pelo Programa de Melhoramento Genético de Citros da Embrapa Mandioca e Fruticultura (PMG Citros), sendo eles: citrandarin Indio, citrandarin Riverside, citrandarin San Diego, tangerineira Sunki Tropical, limoeiro Cravo Santa Cruz, híbrido LVK x LCR - 038, híbrido LVK x LVA - 009, híbrido TSKC x CTSW - 041, híbrido TSKFL x CTTR - 017 e híbrido TSKFL x CTTR - 013.

As mudas foram obtidas em viveiro telado com $50 \%$ de sombreamento pelo método de enxertia por borbulhia em "T" invertido, cuja inserção da borbulha da 
copa foi feita a $30 \mathrm{~cm}$ do colo do porta-enxerto com posterior amarrio através de fita de polietileno para a fixação das partes (GUILHERME et al., 2014; PORTELA et al., 2016; RODRIGUES et al., 2016).

O delineamento experimental adotado foi em blocos ao acaso, com dez tratamentos (porta-enxertos) e três repetições. A parcela foi composta por duas plantas úteis, no espaçamento de 7,0m x 7,0m. Como bordadura externa do experimento, utilizou-se plantas de Limeira ácida Tahiti enxertadas sobre o limoeiro Cravo comum.

O plantio foi realizado em covas com dimensões de $0,40 \times 0,40 \times 0,40 \mathrm{~m}$ (comprimento, largura e profundidade). Os tratos culturais utilizados para a cultura no período de execução do experimento foi de acordo com as recomendações de Mattos Junior et al. (2014) que incluíram correção do solo antes do plantio e adubação no início e final do período de chuvas (novembro e maio). Não foram realizados controles preventivos de pragas na área experimental.

Em 2016, 06 anos após o plantio, entre os meses de maio e junho, foram mensuradas as seguintes características das plantas: Altura $(H)$, medida em metros, do colo até o topo; volume da copa ((V), obtido pela Equação 1.

$$
\mathrm{V}=2 / 3 \pi \mathrm{Dm}^{2} / 4 \times \mathrm{H}
$$

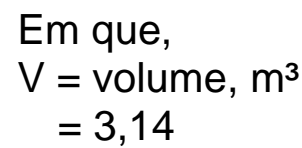

$\mathrm{Dm}(\mathrm{m})=(\mathrm{DI}+\mathrm{Dr}) / 2$, sendo $\mathrm{DI}(\mathrm{m})$ obtida pela medida da copa no sentido da linha e $\operatorname{Dr}(\mathrm{m})$ determinada no sentido perpendicular à rua;

$\mathrm{H}=$ altura da planta $(\mathrm{m})$.

A Eficiência produtiva (EFP), foi calculada a partir da razão entre a produção por planta e o volume da copa $\left(\mathrm{kg} \mathrm{m}^{-3}\right)$; Compatibilidade (COM) através da razão entre os diâmetros do caule do porta-enxerto e enxerto, com auxílio de suta, ambos medidos $10 \mathrm{~cm}$ acima e abaixo do ponto de enxertia; porcentagem de sobrevivênciaPV (\%) calculada pela razão das plantas mortas em relação às vivas até 2016;

Foi determinado o número médio de frutos por planta (NMF), obtido por meio da contagem total de frutos na planta e frutos caídos no chão; produção por planta $\left(\mathrm{kg} \mathrm{pl}^{-1}\right)$, mensurada nas safras de 2015 e 2016, estimada pelo produto das variáveis número médio de fruto por planta e massa média do fruto $(\mathrm{g})$ e produção acumulada $\left(\mathrm{kg} \mathrm{pl}^{-1}\right)$, obtida a partir da soma da produção ao longo das duas safras. Essas variáveis são as mais usuais para avaliar a produção de citros conforme pode ser verificado em outros trabalhos (FRANÇA et al., 2016; SAMPAIO et al., 2016; SANTOS et al., 2016; RAMOS, et al., 2015).

$\mathrm{Na}$ época de amadurecimento, geralmente entre maio e junho, foram colhidos dez frutos por planta, num total de 20 frutos maduros por parcela, num total de 60 por tratamento, e transportados para o laboratório de pós-colheita da Embrapa Acre para ser avaliada a massa média do fruto - MF $(\mathrm{g})$, por pesagem em balança digital dos frutos colhidos; diâmetro do fruto - DF $(\mathrm{mm})$ e comprimento - CF $(\mathrm{mm})$ do fruto e espessura da casca - EC $(\mathrm{mm})$, medidos com paquímetro digital; rendimento de suco - RS (\%) calculado a partir da relação (massa do suco/massa do fruto) x 100; Sólidos solúveis (SS), realizada com um refratômetro digital ( ${ }^{\circ}$ Brix); Acidez titulável (AT), obtida pelo método de titulação com hidróxido de sódio $(\mathrm{NaOH}) 0,1 \mathrm{~N}$, e; Relação SS/AT (ratio). 
As variáveis de qualidade de frutos foram expressas em termos médios das colheitas realizadas no experimento durante os dois anos de avaliação.

Os dados obtidos foram submetidos ao teste de Shapiro-Wilk para verificação da normalidade e ao teste de Cochran para verificar a homogeneidade das variâncias. Após a verificação dos pressupostos, realizou-se a análise de variância e as médias foram agrupadas pelo teste de Scott-Knott, ao nível de 5\% de significância.

\section{RESULTADOS E DISCUSSÃO}

Os porta-enxertos citrandarins Riverside e San Diego, limoeiro Cravo Santa Cruz e os híbridos LVK x LCR - 038, LVK x LVA - 009 e TSKFL x CTTR - 013 levaram as plantas a atingirem maior crescimento, tanto em altura, como em volume de copa, indicando, assim, um maior vigor desses genótipos (Tabela 2).

TABELA 2 - Características* vegetativas e de produção, entre 2015 e 2016, de limeira ácida Tahiti [Citrus latifolia (Yu. Tanaka) Tanaka] enxertada em dez portaenxertos de citros em Capixaba, Acre

\begin{tabular}{|c|c|c|c|c|c|c|}
\hline Porta-enxertos & H (m) & $\operatorname{VC}\left(\mathrm{m}^{3}\right)$ & $\begin{array}{c}\text { EFP } \\
\left(\mathrm{kg} \mathrm{m}^{3}\right)\end{array}$ & COM & PV (\%) & NMF \\
\hline Citrandarin Indio & $3,08 b$ & $28,34 c$ & $1,05 a$ & $0,94 a$ & $83,67 b$ & $249,66 a$ \\
\hline Citrandarin Riverside & $3,78 a$ & $41,53 a$ & $0,91 b$ & $0,92 a$ & $100,00 a$ & $254,76 a$ \\
\hline Citrandarin San Diego & $3,81 a$ & $51,72 a$ & $0,93 b$ & $0,93 a$ & $100,00 a$ & $279,78 a$ \\
\hline Sunki Tropical & $3,11 b$ & $33,21 b$ & $0,74 b$ & $0,93 a$ & $100,00 a$ & $183,00 b$ \\
\hline Cravo Santa Cruz & $3,77 a$ & $48,29 a$ & $0,68 b$ & $0,89 a$ & $53,33 c$ & $265,33 a$ \\
\hline LVK x LCR - 038 & $3,93 a$ & $45,47 a$ & $0,84 b$ & $0,88 a$ & $50,00 c$ & $204,33 b$ \\
\hline LVK x LVA - 009 & $3,70 a$ & $43,23 a$ & $0,81 b$ & $0,87 a$ & $83,33 b$ & $180,57 c$ \\
\hline TSKC x CTSW - 041 & $2,85 b$ & $25,18 c$ & $1,06 a$ & $0,88 a$ & $83,33 b$ & $199,83 b$ \\
\hline TSKFL x CTTR - 017 & $3,55 a$ & $36,00 b$ & $0,70 b$ & $0,93 a$ & $36,67 c$ & $197,50 b$ \\
\hline TSKFL x CTTR - 013 & $3,73 a$ & $43,77 a$ & $0,77 b$ & $0,85 a$ & $83,67 b$ & $180,01 c$ \\
\hline CV (\%) & 12,11 & 12,51 & 24,44 & 15,56 & 39,37 & 16,11 \\
\hline
\end{tabular}

Médias seguidas pela mesma letra, não diferem estatisticamente entre si pelo teste de Scott- Knott a $5 \%$ de probabilidade.

* H: Altura de planta; VC: volume de copa; EFP: eficiência produtiva; COM: relação diâmetro da copa e porta-enxerto; PV: percentagem de sobrevivência e; NMF: número médio de frutos.

A redução da altura foi observada em plantas enxertadas sobre enxertos citrandarin Indio, Sunki Tropical e o híbrido TSKC x CTSW - 041 (Tabela 2) o que permite inferir que o espaçamento adotado para esses porta-enxertos poderia ser menor e, com isso, permitiria o maior adensamento do plantio. Além disso, a redução do porte permite a melhor realização da colheita e aplicação dos tratos culturais (PORTELA et al., 2016).

A eficiência produtiva mede a produção de frutos por volume de copa (LIMA et al., 2014). Quanto maior a produção por volume de copa maiores são as chances de se adotar altas densidades de plantio com consequente aumento de produtividade. Verificou-se que o citrandarin Indio e o híbrido TSKC x CTSW - 041 apresentaram as maiores eficiências na produção (Tabela 2). Nos demais porta-enxertos observou-se baixa eficiência produtiva, o que pode estar relacionada ao maior volume de copa (Tabela 2), pois está variável é inversamente relacionada ao crescimento de copa (FRANÇA et al. 2016). Segundo Bacar et al., (2017), portaenxertos que induzem alta eficiência produtiva e baixo volume de copa são mais 
interessantes que aqueles com alto volume de copa e maior rendimento total de frutos por planta, pois indicam que essa produção pode ser compensada pelo aumento da densidade de plantas por área.

A compatibilidade dos porta-enxertos com a limeira ácida Tahiti foi alta, com a razão do diâmetro do caule do porta-enxerto e do enxerto (RD) superior a 0,80 (Tabela 2), ficando próximo da compatibilidade plena, o que sugere boa afinidade da copa com os porta-enxertos estudados. De acordo com Emmanouilidou e Kyriacou (2017) a compatibilidade entre copa e porta-enxerto é um requisito tão importante quanto os atributos agronômicos e à resistência a estresses bióticos e abióticos para a seleção de porta-enxerto.

A enxertia de limeira ácida Tahiti sobre os porta-enxertos citrandarins Riverside e San Diego e tangerineira Sunki Tropical promoveu 100\% de plantas vivas (sobrevivência) nos seis anos do experimento (Tabela 2). No entanto, observou-se redução do estande de plantas dos genótipos LVK x LCR - 038, TSKFL x CTTR 017 e limoeiro Cravo Santa Cruz (Tabela 2). A morte dessas plantas pode estar relacionada ao ataque de gomose de Phytophthora, já que os sintomas visuais típicos da doença, como clorose, quedas de folhas, rachadura do caule próximo à região de enxertia, secamento completo e, consequente, morte da planta, foram pronunciados.

O maior número de frutos observados foi no induzido pelos porta-enxertos citrandarins Indio, Riverside e San Diego e limoeiro Cravo Santa Cruz (Tabela 2). Já os híbridos LVK x LVA - 009 e TSKFL x CTTR - 013 e a tangerineira Sunki Tropical apresentaram os menores valores, não superando 185 frutos por planta (Tabela 2). Machado et al. (2017) também observaram baixo número de frutos de lima ácida Tahiti sobre tangerineira Sunki.

A produção por planta variou nos dois anos para todos os porta-enxertos, com destaque para os citrandarins Indio, Riverside e San Diego e o limoeiro Cravo Santa Cruz que se mantiveram como os mais produtivos ao longo desse período avaliado (Tabela 3). Estes resultados são superiores aos relatados por Villa et al. (2017) para a cultivar de lima ácida Tahiti IAC-5 enxertada no Trifoliata Flying Dragon aos 4 anos de idade. Por outro lado, os híbridos LVK x LCR - 038, LVK x LVA - 009 e TSKFL x CTTR - 017 foram os menos produtivos (Tabela 3 ).

Os porta-enxertos que levaram a uma maior produção acumulada por planta foram os citrandarins Indio, Riverside e San Diego, além do limoeiro Cravo Santa Cruz e do híbrido LVK x LCR 038 (Tabela 3). A baixa produção por planta se deve, provavelmente, à idade do pomar, pois há incremento gradual de produção dessa espécie ao longo dos anos até atingir a estabilidade produtiva que ocorre por volta do oitavo ano após o plantio.

TABELA 3 - Produção e produção acumulada, entre 2015 e 2016, de limeira ácida Tahiti [Citrus latifolia (Yu. Tanaka) Tanaka] enxertada em dez porta-enxertos de citros em Capixaba - AC 


\begin{tabular}{lccc}
\hline Porta-enxertos & $\begin{array}{c}\text { Produção 2015 } \\
\mathbf{( k g ~ p l}^{-1} \mathbf{)}\end{array}$ & $\begin{array}{c}\text { Produção 2016 } \\
\mathbf{( k g ~ p l}^{-1} \mathbf{)}\end{array}$ & $\begin{array}{c}\text { Produção } \\
\text { acumulada } \\
\mathbf{( k g ~ p ~}^{-1} \mathbf{)}\end{array}$ \\
\hline Citrandarin Indio & $22,52 \mathrm{a}$ & $17,42 \mathrm{a}$ & $39,94 \mathrm{a}$ \\
Citrandarin Riverside & $20,67 \mathrm{a}$ & $15,07 \mathrm{a}$ & $35,74 \mathrm{a}$ \\
Citrandarin San Diego & $19,41 \mathrm{a}$ & $18,83 \mathrm{a}$ & $38,24 \mathrm{a}$ \\
Sunki Tropical & $15,90 \mathrm{a}$ & $12,04 \mathrm{~b}$ & $27,94 \mathrm{c}$ \\
Cravo Santa Cruz & $21,30 \mathrm{a}$ & $15,31 \mathrm{a}$ & $36,61 \mathrm{a}$ \\
LVK x LCR - 038 & $23,68 \mathrm{a}$ & $13,07 \mathrm{~b}$ & $36,75 \mathrm{a}$ \\
LVK x LVA - 009 & $12,43 \mathrm{~b}$ & $13,36 \mathrm{~b}$ & $25,79 \mathrm{c}$ \\
TSKC x CTSW - 041 & $14,56 \mathrm{~b}$ & $17,80 \mathrm{a}$ & $32,36 \mathrm{~b}$ \\
TSKFL x CTTR - 017 & $13,22 \mathrm{~b}$ & $12,93 \mathrm{~b}$ & $26,15 \mathrm{c}$ \\
TSKFL x CTTR - 013 & $14,70 \mathrm{~b}$ & $16,21 \mathrm{a}$ & $30,91 \mathrm{~b}$ \\
\hline CV (\%) & $\mathbf{1 6 , 7 3}$ & $\mathbf{1 9 , 4 7}$ & $\mathbf{1 3 , 6 7}$ \\
\hline
\end{tabular}

Médias seguidas pela mesma letra não diferem estatisticamente entre si pelo teste de ScottKnott a $5 \%$ de probabilidade.

Os porta-enxertos citrandarins Indio e San Diego, Sunki Tropical e o limoeiro Cravo Santa Cruz formaram o grupo dos genótipos que induziram as maiores massas médias dos frutos, 108,23g, 101,99g, 101,67g e 102,74g, respectivamente (Tabela 4), semelhantes às observadas por Santos et al. (2016) em diferentes seleções de Tahiti sobre citrumelo Swingle e, superiores às relatadas por Simões et al. (2017), onde a massa do fruto de Tahiti sobre limoeiro Cravo variou de 80,21 a 87,25 g. O tamanho e a massa dos frutos estão diretamente relacionadas à disponibilidade hídrica do solo e, por isso, acredita-se os resultados observados nesse estudo reforçam tal hipótese. Outra questão que merece destaque diz respeito ao material genético de Tahiti utilizado.

Quanto ao diâmetro e comprimento do fruto, não foi observada diferença entre os porta-enxertos (Tabela 4). Foram verificadas médias variando entre 52,98 mm (TSKFL x CTSW 041) e 64,51 mm (limoeiro Cravo Santa Cruz) para diâmetro e 52,76 $\mathrm{mm}$ (TSKFL x CTTR 017) e 67,39 mm (tangerineira Sunki Tropical) para comprimento. Alves-Júnior et al. (2011) e Nascente et al. (2015) também não detectaram diferenças para essas variáveis, mas os valores foram inferiores aos deste estudo.

Os porta-enxertos citrandarin Riverside, tangerineira Sunki Tropical, limoeiro Cravo Santa Cruz e os híbridos LVK x LCR - 038 e TSKC x CTSW - 041 conferiram a formação de frutos com menor espessura de casca (Tabela 4). De acordo com os atacadistas da Central de Abastecimento do Estado de São Paulo - CEAGESP (2011), o mercado interno prefere lima ácida Tahiti com casca lisa, fina com muito suco e o mercado externo prefere frutas com casca verde intensa.

Para o rendimento de suco, não houve diferença entre os porta-enxertos (Tabela 4). Segundo os padrões de classificação de lima ácida Tahiti, os frutos para exportação devem apresentar no mínimo $40,0 \%$ de porcentagem de suco (CEAGESP, 2011). Critério atendido por todos os porta-enxertos estudados em combinação com a limeira ácida Tahiti.

TABELA 4 - Características* físicas, químicas e físico-químicas de frutos, entre 2015 e 2016, de lima ácida Tahiti [Citrus latifolia (Yu. Tanaka) Tanaka] enxertada em dez porta-enxertos de citros, em Capixaba - AC 


\begin{tabular}{|c|c|c|c|c|c|c|c|c|}
\hline Porta-enxertos & MF (g) & $\begin{array}{c}\text { DF } \\
(\mathrm{mm})\end{array}$ & $\begin{array}{c}\text { CF } \\
(\mathrm{mm})\end{array}$ & $\begin{array}{c}\text { EC } \\
(\mathrm{mm})\end{array}$ & $\begin{array}{l}\text { RS } \\
\text { (\%) }\end{array}$ & $\begin{array}{c}\text { SS } \\
\left({ }^{\circ} \text { Brix }\right)\end{array}$ & $\begin{array}{l}\text { AT } \\
(\%)\end{array}$ & RATIO \\
\hline Citrandarin Indio & $108,23 a$ & $59,13 a$ & $57,48 a$ & $3,94 a$ & $49,84 a$ & $7,66 a$ & $6,00 a$ & $1,28 a$ \\
\hline Citrandarin Riverside & $85,19 b$ & $62,06 \mathrm{a}$ & $53,53 a$ & $2,98 b$ & $52,64 a$ & $7,88 a$ & $5,83 b$ & $1,35 a$ \\
\hline Citrandarin San Diego & $101,99 a$ & $57,18 a$ & $54,24 a$ & $3,72 a$ & $55,24 a$ & $7,56 a$ & $6,33 a$ & $1,19 a$ \\
\hline Sunki Tropical & $101,67 a$ & $61,20 a$ & $67,39 a$ & $2,62 b$ & $55,89 a$ & $7,78 a$ & $6,03 a$ & $1,23 a$ \\
\hline Cravo Santa Cruz & $102,74 a$ & $64,51 \mathrm{a}$ & $61,95 a$ & $2,40 b$ & $56,05 a$ & $7,86 a$ & $6,10 a$ & $1,28 a$ \\
\hline LVK x LCR - 038 & $89,95 b$ & $55,61 a$ & $53,16 a$ & $2,90 b$ & $48,19 a$ & $7,86 a$ & $5,67 b$ & $1,38 a$ \\
\hline LVK x LVA - 009 & $83,63 b$ & $53,45 a$ & $55,40 a$ & $3,22 a$ & $49,47 a$ & $7,00 \mathrm{~b}$ & $5,80 b$ & $1,20 \mathrm{a}$ \\
\hline TSKC x CTSW - 041 & $86,69 b$ & $52,98 a$ & $56,92 a$ & $2,43 b$ & $57,71 a$ & $7,63 a$ & $5,84 b$ & $1,30 a$ \\
\hline TSKFL x CTTR - 017 & $84,76 b$ & $54,66 a$ & $52,76 a$ & $3,16 a$ & $47,93 a$ & $7,75 a$ & $5,85 b$ & $1,32 \mathrm{a}$ \\
\hline TSKFL x CTTR - 013 & $91,53 \mathrm{~b}$ & $56,00 a$ & $53,01 \mathrm{a}$ & $3,42 a$ & $51,69 a$ & $7,02 b$ & $5,80 b$ & $1,21 \mathrm{a}$ \\
\hline Média & 93,64 & 57,68 & 56,58 & 3,08 & 52,47 & 7,60 & 5,93 & 1,27 \\
\hline CV (\%) & 20,01 & 10,63 & 12,11 & 16,12 & 10,80 & 2,92 & 5,62 & 5,08 \\
\hline
\end{tabular}

Médias seguidas pela mesma letra, não diferem estatisticamente entre si pelo teste de Scott- Knott a $5 \%$ de probabilidade.

* MF: massa de fruto; DF: diâmetro do fruto; CF: comprimento do fruto; EC: espessura da casca; RS: rendimento de suco; SS: sólidos solúveis; AT: acidez titulável.

A copa de limeira ácida Tahiti enxertada nos porta-enxertos citrandarins Indio, Riverside e San Diego, bem como na tangerineira Sunki Tropical, no limoeiro Cravo Santa Cruz e nos híbridos LVK x LCR - 038, TSKC x CTSW - 041 e TSKFL x CTTR 017 produziu frutos com maior teor de sólidos solúveis (Tabela 4).

A concentração de SS verificada neste trabalho é inferior às observadas em outros trabalhos (BREMER NETO et al., 2013; CANTUARIAS-AVILÉS et al., 2012), - que pode ser explicado pela precipitação ocorrida na região (Figura 1) no momento da maturação dos frutos, já que altas precipitações na época de crescimento e maturação dos frutos influencia negativamente a concentração sólidos solúveis.

Para a acidez titulável, os frutos mais ácidos foram observados nas plantas enxertadas sobre os citrandarins Indio $(6,00 \%)$ e San Diego $(6,33 \%)$, na tangerineira Sunki Tropical $(6,03 \%)$ e no limoeiro Cravo Santa Cruz (6,10\%) (Tabela 4). Estes resultados são inferiores aos obtidos por Nascente et al. (2015) para a lima ácida Tahiti com três anos de idade enxertada no limoeiro Cravo. Isso pode estar relacionado à temperatura, já que é o fator que mais influência no acúmulo do ácido cítrico que, ao alcançar o valor máximo, diminui devido ao aumento ao tamanho do fruto e sua utilização no processo respiratório (SIMONETTI et al., 2015). Temperaturas elevadas (Figura 1) durante a fase de maturação podem explicar a menor acidez observada nos frutos.

A ratio dos frutos da limeira ácida Tahiti, variável que mede o índice de maturação dos frutos, não sofreu influência dos porta-enxertos (Tabela 4), resultado similar ao detectado por Santos et al. (2016).

\section{CONCLUSÕES}

Houve efeito dos porta-enxertos sobre o comportamento da variedade copa de limeira ácida Tahiti.

As plantas da limeira ácida Tahiti apresentaram menor porte e maior eficiência produtiva quando enxertadas nos porta-enxertos citrandarin Indio e híbrido TSKC $\mathrm{x}$ CTSW -041.

Os porta-enxertos citrandarins Indio, Riverside e San Diego, bem como o limoeiro Cravo Santa Cruz induzem maior produção da copa da limeira ácida Tahiti.

Os citrandarins Indio, Riverside e San Diego, tangerineira Sunki Tropical e 
limoeiro Cravo Santa Cruz proporcionaram frutos de lima ácida de melhor qualidade.

\section{AGRADECIMENTOS}

À Coordenação de Aperfeiçoamento de Pessoal de Nível Superior (CAPES) pela bolsa de doutorado; à Embrapa Mandioca e Fruticultura pelo apoio e fornecimento de materiais genéticos (porta-enxertos); à Embrapa Acre pela infraestrutura física e pessoal; à Universidade Federal do Acre por dar a oportunidade de ser realizado o doutoramento do primeiro autor em produção vegetal; e ao produtor, Sr. Barroso, pela parceria para execução da pesquisa.

\section{REFERÊNCIAS}

AGRITEMPO. Sistema de monitoramento agrometeorológico. Disponível em < https://www.agritempo.gov.br/agritempo/index.jsp> Acesso em: 06 ago. de 2018.

ALVES-JÚNIOR, J.; FOLEGATTI, M.V.; SILVA, C.R. da. Response of young Tahiti lime trees to diferente irrigation levels. Engenharia Agrícola, v. 31, n. 02, p. $303-$ 314, 2011. Disponível em: < http://dx.doi.org/10.1590/S0100-69162011000200010>.

BACAR, E. L. C.; NEVES, C. S. V J.; LEITE JUNIOR, R. P.; YADA, I. F. U.; TAZIMA, Z. H. 'JAFFA' SWEET ORANGE PLANTS GRAFTED ONTO FIVE ROOTSTOCKS. Revista Brasileira de Fruticultura, v.39, p. 200, 2017. Disponível em: $<$ http://dx.doi.org/10.1590/0100-29452017200>.

BREMER NETO, H.; MOURÃO FILHO, F.A.A.; STUCHI, E.S.; ESPINOZA-NÚÑEZ, E.; CANTUARIAS-AVILÉS, T. The horticultural performance of five Tahiti lime selections grafted onto 'Swingle' citrumelo under irrigated and non-irrigated conditions. Scientia Horticulturae, v. 150, p.181-186, 2013. Disponível em: < https://doi.org/10.1016/j.scienta.2012.10.010>.

CANTUARIAS-AVILÉS, T.; MOURÃO FILHO, F.A.A.; STUCHI, E.S.; SILVA, S.R.; ESPINOZA-NÚÑEZ, E.; BREMER NETO, $H$. Rootstocks for high fruit yield and quality of Tahiti lime under rain-fed conditions. Scientia Horticulturae, v. 142, p.105111, 2012. Disponível em: <http://dx.doi.org/10.1016/j.scienta.2012.05.008>.

CARVALHO, H. W. L.; MARTINS, C. R.; TEODORO, A. V.; SOARES FILHO, W. S.; PASSOS, O. S. (2016). Agronomical performance of 'Piemonte' mandarin grafted on several rootstocks in the Brazilian Coastal Tablelands. Pesquisa Agropecuária Brasileira, v.51, n.11, p.1830-1838, 2018. Disponível em: DOI: 10.1590/S0100204X2016001100005

CEAGESP. Companhia de Entrepostos Armazéns Gerias do Estado de São Paulo. Classificação do limão (lima ácida) Tahiti (Citrus latifolia Tanaka). São Paulo, 2011. Disponível em: <www.ceagesp.com.br>. Acesso em: 18 ago. 2018.

EMMANOUILIDOU, M.G.; KYRIACOU, M.C. Rootstock-modulated yield performance, fruit maturation and phytochemical quality of Lane Late' and 'Delta'sweet orange. Scientia Horticulturae, v. 225, p. 112-121. 2017. Disponível em: <https://doi.org/10.1016/j.scienta.2017.06.056>.

FAO. [Produtividade por país]. Disponível em: <http://faostat.fao.org>. Acesso em: 24 junho. 2018. 
FRANÇA, N. O.; AMORIM, M. S.; GIRARDI, E. A.; PASSOS, O. S.; SOARES FILHO, W. dos S. Performance of 'Tuxpan Valencia' sweet orange grafted onto 14 rootstocks in northern Bahia, Brazil. Revista Brasileira de Fruticultura, v.38, e-684, 2016. Disponível em: <http://dx.doi.org/10.1590/0100-29452016684>.

GUILHERME, D. O.; MARINHO, C. S.; BIAZATT, M. A.; CAMPOS, G. S. E.; BREMENKAMP, C. A. Produção de mudas de laranjeira Pêra por meio do método de interenxertia. Ciência Rural, v. 44, p. 414-417, 2014. Disponível em: < http://dx.doi.org/10.1590/S0103-84782014000300005>.

IBGE - Instituto Brasileiro de Geografia e Estatística. Estatísticas sobre produção agrícola municipal. [2018]. Disponível em: <www.sidra.ibge. gov.br> Acesso em: 18 ago. 2018.

IBRAF - Instituto Brasileiro de Frutas. Exportações de frutas frescas-2015. Disponível em: <http://www.ibraf.org.br/estatisticas/est_frutas.asp>. Acesso em: 10/08/2018.

LEDO, A. da S.; OLIVEIRA, T. K. de; RITZINGER, R. AZEVEDO, F. F. de. Produção de limas ácidas, tangerineira e híbridos sobre diferentes porta-enxertos no Estado do Acre. Revista Ciência Agronômica, Fortaleza, v. 39, n. 02, p. 263-268, 2008.

LIMA, C.F. de; MARINHO, C. S.; COSTA, E. S.; ALMEIDA, T. R. de V.; AMARAL, C. $O$. Qualidade dos frutos e eficiencia produtiva da laranjeira 'Lima' enxertada sobre Trifloliata', em cultivo irrigado. Revista Brasileira de Ciências Agrárias, v. 9, n. 3, p. 401 - 405, 2014. Disponível em: <http://www.redalyc.org/articulo.oa?id=119032103014>.

MACHADO, D. L. M.; SIQUEIRA, D. L. de; SALOMÃO, L. C. C.; CECON, P. R.; SILVA, D. F. P. da. Evaluation of rootstocks for Tahiti acid lime In northern state of Minas Gerais. Revista Brasileira de Fruticultura, Jaboticabal, v. 39, n. 1, p. 778790, 2017. Disponível em: <http://dx.doi.org/10.1590/0100-29452017790 >. DOI: 10.1590/0100-29452017 790 .

MATTOS JUNIOR, D.; NEGRI, J. D. de; POMPEU JUNIOR, J.; GHILARDI, A. A.; AZEVEDO, F. A. de; BASTIANEL, M. Citros: principais informações e recomendações de cultivo. In: AGUIAR, A. T. da E.; GONÇALVES, C.; PATERNIANI, M. E. A. G. Z.; CARLOS, M. L. S. T. (Eds.). Instruções Agrícolas para as Principais Culturas Econômicas. 7. ed. Campinas: Instituto Agronômico, 2014, p. 140-149 (Boletim, 200).

NASCENTE, A. C. S.; ANJOS, R. A. R.; SANTOS, P. G. F.; MENDES, L. W. A.; CUSTÓDIO, J. P. C.; DION, H. Jr.; SILVEIRA, P. S.; MATOS, F. S. Productivity of Citrus latifolia plants subjected to branch girdling. African Journal of Agricultural Research, v.10, n.46, p.4209-4214, 2015. Disponível em: < http://www.academicjournals.org/AJAR>. DOI: 10.5897/AJAR2015.10391.

PORTELLA, C. R.; MARINHO, C. S.; AMARAL, B. D.; CARVALHO, W. S. G.; CAMPOS, G. S.; SILVA, M. P. S. da; SOUSA, M. C. de. Desempenho de cultivares de citros enxertadas sobre o trifoliateiro 'Flying Dragon' e limoeiro 'Cravo' em fase de formação do pomar. Bragantia, v. 75, p.70-75, 2016. Disponível em: < http://dx.doi.org/10.1590/1678-4499.267>. 
RAMOS, Y. C.; STUCHI, E. S.; GIRARDI, E. A.; LEÃO, H. C. de; GESTEIRA, A. da S.; PASSOS, O. S.; SOARES FILHO, W. dos S. Dwarfing rootstocks for 'Valencia' sweet orange. Acta Horticulturae, v. 1065, p. 351-354, 2015. Disponível em: <http://dx.doi.org/10.17660/ActaHortic.2015.1065.42>.

RODRIGUES, M. J. da S.; OLIVEIRA, E. R. M. de; GIRARDI, E. A.; LEDO, C. A. da S.; SOARES FILHO, W. dos S. Produção de mudas de citros com diferentes combinações copa e porta-enxerto em viveiro protegido. Revista Brasileira de Fruticultura, Jaboticabal, v.38, n. 1. P. 187-201, Fev. 2016. Disponível em: < http://dx.doi.org/10.1590/0100-2945-284/14>.

SAMPAIO, A. H. R.; COELHO FILHO, M. A.; SOUZA, L. D.; BRITO, R. B. F.; SILVA, R. O. da S. Rield and quality of 'Pera' sweet orange grafted on different rootstocks under rainfed conditions. Revista Brasileira de Fruticultura, Jaboticabal, v. 38, n. 3, p. 770-779, mai/jun 2016. Disponível em: < http://dx.doi.org/10.1590/010029452016770>.

SANTOS, M. G. dos; SOARES FILHO, W. dos S.; GIRARDI, E. A.; GESTEIRA, A. da S.; PASSOS, O. S.; FERREIRA, C. F. Initial horticultural performance of nine 'Persian' lime selections grafted onto Swingle citrumelo. Scientia Agricola, v.73, n.2, p.109-114, Mar/Abr 2016. Disponível em: < http://dx.doi.org/10.1590/0103-90162015-0058>.

SIMÕES, W. L.; COELHO, E. F.; MARTINEZ, M. A.; COELHO FILHO, M. A.; COSTA, E. L. da; GOMES, V. H. Produtividade e características físico-químicas dos frutos da lima ácida Tahiti' sob diferentes disposições de microaspersores. Water Resources and Irrigation Management, Cruz das Almas, v.6, n.1, p.107-114, 2017.

SIMONETTI, L. M.; CRISTOFANI-YALY, M.; BARROS, V. L. N. P. de; SCHINOR, E. H.; FADEL, A. L.; SOUSA, M. C.; LEONEL, S.; TECCHIO, M. A. Porta-enxertos alternativos para cultivo de laranja Valência na região sudoeste do estado de São Paulo. Citrus Research \& Technology, v.36, n.2, p.49-58, 2015. Disponível em: $<$ http://dx.doi.org/10.4322/crt08916>.

VILLA, F.; FARIA, F. B.; KLOSOWSKI, E. S.; TSUTSUMI, C. Y. Produtividade e fenologia de lima ácida Tahiti em região subtropical de baixa altitude do Paraná. Scientia Agraria Paranaensis, Marechal Cândido Rondon, v. 16, n. 2, abr./jun., p. 171-178, 2017. Disponível em: < http://dx.doi.org/10.18188/19831471/sap.v16n1p171-178>. 